TITLE:

\title{
Modeling thermal behaviors of window flame ejected from a fire compartment
}

\section{AUTHOR(S):}

HIMOTO, Keisuke; TSUCHIHASHI, Tsuneto; TANAKA, Yoshiaki; TANAKA, Takeyoshi

\section{CITATION:}

HIMOTO, Keisuke ... [et al]. Modeling thermal behaviors of window flame ejected from a fire compartment. Fire Safety Journal 2009, 44(2): 230240

\section{ISSUE DATE:}

2009-02

URL:

http://hdl.handle.net/2433/93450

\section{RIGHT:}

Copyright @ 2008 Elsevier; この論文は出版社版でありません。引用の 際には出版社版をご確認ご利用ください。; This is not the published version. Please cite only the published version. 


\section{Modeling Thermal Behaviors of}

\section{Window Flame Ejected from A Fire Compartment}

Keisuke HIMOTO (corresponding author)

Pioneering Research Unit for Next Generation, Kyoto Univ.

Gokasho, Uji, Kyoto 611-0011, Japan

e-mail keisuke.himoto@kupru.iae.kyoto-u.ac.jp

tel. $\quad+81-774-38-4481$

fax $+81-774-38-4039$

Tsuneto TSUCHIHASHI, and Yoshiaki TANAKA

General Building Research Corp. of Japan

Fujishirodai 5-8-1, Suita, Osaka 565-0873, Japan

Takeyoshi TANAKA

Disaster Prevention Research Institute, Kyoto Univ.

Gokasho, Uji, Kyoto 611-0011, Japan

\section{ABSTRACT}

A series of reduced-scale experiments were carried out in order to investigate thermal behaviors of window flame, which exposes the upper floors as well as the adjacent buildings to potential 
risks of fire spread. A stainless pan filled with alcohol was used as the fire source and was

placed inside a cubic compartment of $900 \mathrm{~mm}$ side. Temperatures and velocities at various points inside and outside of the compartment were measured. The compartment was pressurized during the experiment by mechanically supplying air at several mass inflow rates through an inlet duct set at the bottom part of the compartment. This was for simulating fire conditions under the effect of external wind pressure. Based on the experimental observation, line (i.e., two dimensional) heat source assumption was adopted for developing a model of window flame behavior. A dimensionless parameter $Q^{\prime *}$ was derived from the governing differential equations, in order to generalize the measurement results. Expressions for temperature rise along the trajectory $\Delta T_{m}$ and characteristic flame width $b_{T}$ were developed incorporating the parameter $Q^{\prime *}$.

Keywords: fire spread; window flame; temperature rise; reduced-scale experiment; scale model

\section{INTRODUCTION}

When a fire inside a compartment develops to be at its most vigorous stage, flames would be ejected from the opening after the failure of window glass. Heat flux transferred from the window flames may cause ignition of combustibles stored in rooms of upper floors and/or those 
stored in adjacent buildings. It is generally recognized that window flame is one of the most important contributors of fire spread beyond the room of fire origin. There are already several works done on this topic [1-10], and the knowledge achieved in these works, not merely applied to practice of the building fire safety design, but also give valuable insight into understanding its physical characteristics.

However, we can point out two aspects at which our knowledge is still insufficient and need additional investigation, i.e. : 1) Direction of momentum of window flame is generally horizontal when ejected from an opening. The direction gradually changes into upward due to buoyancy as the initial momentum decreases by entraining quiescent ambient air as getting apart from the opening. However, expressions originally developed for vertically ascending plumes are commonly adopted in generalizing window flame behaviors, and the inherent characteristic of window flame itself is still unexplained; 2) Ventilation behavior of a building in actual urban area is affected by external wind, and so is the window flame behavior when the building is on fire. However, most of the existing burn tests were carried out using compartments with a single opening on one of the vertical walls. In such a condition, window flame behavior is governed either by configuration of the compartment or characteristics of the combustibles. The Effect of external wind needs to be considered in window flame experiments.

In this study, we investigated into thermal behaviors of the window flame experimentally by 
using a reduced-scale compartment. In the experiment, the compartment was pressurized

mechanically at several mass inflow rates in order to simulate variable fire conditions under the effect of external wind. Furthermore, we derived a scaling parameter by analyzing dimensional relations within the governing equations. Expressions for temperature rise along flame trajectory and characteristic flame width were proposed by generalizing the experimental results with the obtained dimensionless parameters.

\section{REDUCED-SCALE EXPERIMENT}

\subsection{Experimental Setup}

Schematic diagram of the model compartment is shown in Fig.1. The compartment, which was constructed with 20mm thick asbestos-perlite boards, was cubic with sides of $900 \mathrm{~mm}$. An opening and an inlet for mechanical air supply were equipped with on the opposing sides of the compartment. The dimension of the opening was changeable in order to set up different fire conditions with regard to opening flow. On the other hand, the air inlet was set at the bottom part of the compartment and the dimension was fixed with 50mm height and 900mm wide, while it was completely shut for no air supply conditions. It was so designed that the stream of the introduced air having upward momentum and avoiding the air to be directly released out of the compartment without mixing. The inlet was connected with a cylindrical duct of $400 \mathrm{~mm}$ 
diameter and a mechanical fan at the other end.

Either methanol or ethanol in a stainless pan was used for the fuel. The amount of alcohol used at individual burn tests was between 3,000 to 4,000ml. The pan was box-shaped at which the depth was $70 \mathrm{~mm}$ and the burn area was $225 \mathrm{~mm}$ by $300 \mathrm{~mm}$. The average duration of the burn tests was $31 \mathrm{~min} 21 \mathrm{sec}$.

An overview of the experiment is shown in Table 1. The configuration of the opening and the mass inflow rate of the mechanical ventilation were varied. As a result, burn tests were carried out at 20 different conditions. The opening width $B$ was changed between 0.2 and $0.5 \mathrm{~m}$, and for the height $H$, between 0.3 and $0.5 \mathrm{~m}$. The corresponding ventilation factor $A \sqrt{H}$ varied from 0.025 to $0.088 \mathrm{~m}^{5 / 2}$. Mass inflow rate by the mechanical fan $\dot{m}_{0}$ was estimated by measuring the flow velocity in the center of an orifice of $70 \mathrm{~mm}$ diameter, which was installed in the introductory part of the cylindrical duct. The measured inflow rate was stable throughout the burn tests and no adverse current was observed.

\subsection{Measurement Data}

Positions of thermocouples for temperature measurement are also shown in Fig.1. A three dimensional orthogonal coordinate system was employed for the use of later analyses: The origin is at the top edge of the opening; $x$-axis perpendicular to the opening plane; $y$-axis parallel to the opening plane; and $z$-axis in the vertical direction. 
For the measurement of temperature inside the compartment, $1.6 \mathrm{~mm}$ diameter sheathed thermocouples were aligned in 3 vertical lines (i.e., in the ventilated side, in the middle and in the window side) with 8 measurement points each. As for the temperatures within the opening plane, 9 measurement points of different heights were aligned on the central axis of the opening, i.e., $y=0$. For the measurement of opening jet temperature outside the compartment, $1.6 \mathrm{~mm}$ diameter K-type thermocouples were aligned on 10 vertical lines with 12 measurement points each. These were tied to a rigid steel mesh so that the positions of the measurement points do not shift during the burn tests. The measured temperatures were recorded with a data-logger at an interval of 30 seconds after the ignition. As the aim of this study was to investigate the behavior at steady state fire condition, data acquired between 10 minutes after ignition and 5 minutes before burnout were used to obtain the steady state temperatures. The average duration of the sampling time period was 14 minutes 30 seconds, and it was observed that the measured temperatures were reasonably stable throughout the periods in every tests.

Bidirectional pitot tubes were used for the flow velocity measurement in the window plane. The positions of the measurement points were the same as those of the temperature measurement, i.e., 9 measurement points of different heights along the vertical axis. Acquisition of the data was started 10 minutes after ignition at time interval of 2 seconds. Steady state velocities were obtained by averaging these data acquired in consecutive time period of 30 seconds. The mass 
flow rate at the opening plane $\dot{m}$ can be evaluated by integrating the flow velocity over the entire opening area (yz-plane). However, as the durations of burn tests were restricted, number of measurement points was not enough to acquire the flow profiles in this way. Thus, the mass flow rate $\dot{m}$ was evaluated by integrating the flow velocities measured at the central line of the opening plane $(y=0)$ in vertical direction, $\dot{m}=\sum_{i}\left(\phi \cdot \rho_{i} u_{i} B \Delta Z_{i}\right)$

where $\rho$ is the flow density, $u$ is the average flow velocity, $\Delta Z$ is the separation of the measurement points, and the suffix $i$ identifies the measurement point. The constant $\phi$ converts the flow velocity at $y=0$ measured with pitot tubes into spatially averaged velocity of the relevant height. The value of $\phi$ were determined from the measured flow profile in the line of $z=-10 \mathrm{~mm}$, which was the only profile measured in the widthwise direction.

The mass loss rate of the fuel was measured by 3 load cells installed beneath the pan. The load cells were sandwiched in two $10 \mathrm{~mm}$-calcium silicate boards, so to avoid loading irregularity. Also, a sheet of ceramic fire blanket was matted over the compartment floor in order to avoid the air leakage through the gap between the floor and loading board. The rest of the gaps of the potential air leakage, such as the member board junctions, were plastered with ceramic fiber paste. Another important parameter to be measured was the virtual heat release rate of window flame 
$\dot{Q}$, which is the sum of the enthalpy ejection rate of hot current through the window $\dot{Q}_{V}$ and heat release rate of flammable gas combustion outside of the compartment $\dot{Q}_{F}$. This value is not congruent to the overall heat release rate of combustion $\Delta H_{F} \dot{m}_{F}$, which could be measured either by the mass loss rate of alcohol fuel or by the oxygen calorimetry. In order to estimate the value of $\dot{Q}$, following conservation equation of energy in quasi-steady fire condition was considered,

$\Delta H_{F} \dot{m}_{F}=\dot{Q}_{W}+\dot{Q}_{D}+\dot{Q}_{V}+\dot{Q}_{F}$

where $\Delta H_{F}$ is the heat of combustion of the gasified fuel, $\dot{m}_{F}$ is the mass loss rate of the fuel, $\dot{Q}_{\text {w }}$ is the heat loss rate to the wall, and $\dot{Q}_{D}$ is the heat loss rate through the opening by radiation. From Eqn. (2), the heat release rate of the window flame $\dot{Q}$ is given by, $\dot{Q}=\dot{Q}_{V}+\dot{Q}_{F}=\Delta H_{F} \dot{m}_{F}-\left(\dot{Q}_{W}+\dot{Q}_{D}\right)$

where $\Delta H_{F} \dot{m}_{F}$ is calculated from the mass loss rate of the alcohol fuel. Assuming that $\dot{Q}_{W}$ is identical to the rate of heat transfer through the wall, and that the compartment gas is black and its emissivity is 1 for $\dot{Q}_{D}$ calculation, we obtain, $\dot{Q}_{W}=\left\{\frac{k}{l}\left(T-T_{\infty}\right)\right\} A_{w}, \quad \dot{Q}_{D}=\sigma\left(T^{4}-T_{\infty}^{4}\right) A_{D}$

where $k$ is the heat conductivity of the material, $l$ is the material width, $\sigma$ is the Stefan-Boltzmann constant, $A_{w}$ is the wall surface area, and $A_{D}$ is the opening area. 


\subsection{Experimental Results and Discussion}

The relationship between the mass supply rate by the fan $\dot{m}_{0}$, and the virtual heat release rate of the window flame $\dot{Q}$, is shown in Fig.2. When the inlet duct was shut and no air was supplied into the compartment ( $\dot{m}_{0}=0$ ), the virtual heat release rate $\dot{Q}$ became the largest when the window was $B=0.2 \mathrm{~m}$ and $H=0.5 \mathrm{~m}$ among the five different window dimensions. However, with this specific window dimensions, $\dot{Q}$ decreased along with the increase of $\dot{m}_{0}$. Contrarily, $\dot{Q}$ increased along with the increase of $\dot{m}_{0}$, for the rest of the opening dimensions.

The relationship between the air supply rate $\dot{m}_{0}$ and compartment gas temperatures at two different opening dimensions is shown in Fig.3. In the both cases, temperatures were almost uniform in the hot gas layers developed under the ceiling. The difference in the measured temperatures, between those above the fuel pan and those of the other positions, is because the thermocouples above the fuel pan were directly exposed to the flame. The tests for $B=0.2 \mathrm{~m}$, $H=0.5 \mathrm{~m}$ were the only examples at which the compartment temperatures decreased due to the mechanical ventilation. This is consistent with the results obtained for the heat release rate $\dot{Q}$ in Fig.2. It is commonly acknowledged that there are two major influences of the air supply on compartment fire behavior, which are intensification of fuel combustion and dilution of compartment gas. These have inverse effect on the compartment gas temperature, i.e., the former raises the gas temperature, whereas the latter reduces the gas temperature. There should 
be a characteristic fuel-air ratio for the most efficient compartment gas combustion, i.e., when compartment gas burns most vigorously. Combustion is intensified by the mechanical air supply when fuel-air ratio approaches the characteristic value, and is attenuated when recedes from it. For example, when a fire is strongly ventilation-controlled, i.e., fuel-air ratio is higher than the characteristic value, combustion intensification effect would prevail with further air supply. On the other hand, when a fire is either weakly ventilation-controlled or fuel-controlled, i.e., when fuel-air ratio is lower than the characteristic value, compartment gas dilution effect would prevail with further air supply. Which of these effects becomes dominant is governed not only by the rate of air supply, but also by various burn conditions such as compartment configuration or properties of combustible upon which the characteristic fuel-air ratio is dependent. The results in Fig. 3 show that the dilution effect of compartment gas was significant when $B=0.2 \mathrm{~m}$, $H=0.5 \mathrm{~m}$, whereas the intensification effect of combustion was significant when $B=0.3 \mathrm{~m}$, $H=0.3 \mathrm{~m}$.

In this study, we defined the window flame trajectory as a line sequentially connecting points of the largest temperature rise at each height. Fig.4 shows the relationship between the temperature rise of the window flame $\Delta T_{m}$ and the separation along the trajectory $\xi$. Excess temperatures $\Delta T_{m}$ were almost constant when separations $\xi$ were small, and they gradually decreased with increase of $\xi$. This general characteristic was common within all of the burn conditions. 
However, magnitudes of temperature rise $\Delta T_{m}$ are more or less grouped by the opening dimension, whereas the effect of air supply was less dominant.

Temperature rises $\Delta T$ and trajectory configurations at different air supply rates $\dot{m}_{0}$ are shown in Fig.5 ((A) is for $B=0.2 \mathrm{~m}, \quad H=0.5 \mathrm{~m}$, and 5(B) is for $B=0.3 \mathrm{~m}, \quad H=0.3 \mathrm{~m})$. When fresh air was introduced by the fan, trajectories receded from walls regardless of opening dimensions. Figures 6 and 7show the profiles of flow velocity and gas temperature at the opening plane, respectively, which are corresponding to burn conditions in Fig.5. Figure 6 shows the descent of neutral plane heights with mechanical air supply, which is due to the increase of pressure inside compartment. There is also increase in the maximum velocities of vent flows with increasing pressure, which in turn, yields increase in initial momentum of window flame at ejection.

Trajectory attachment to the wall was observed when $B=0.3 \mathrm{~m}, \quad H=0.3 \mathrm{~m}$, and $\dot{m}_{0}=0$. One of the important reasons suggested by Yokoi is the pressure difference between the wall-side and open-side of window flame. This is due to the restriction of air entrainment from the wall-side of window flame by the presence of wall [1]. Yokoi also pointed out that attachment is prone to occur when $B$ is wide [1]. This observation is partly consistent with the present results in that the attachment was observed when $B=0.3 \mathrm{~m}$, while not when $B=0.2 \mathrm{~m}$. However, it is also clear that window width $B$ is not the only contributing factor, as flow attachment was not 
observed when mechanically ventilated even though $B=0.3 \mathrm{~m}$.

\section{SCALE MODELING}

\subsection{Outline of the Model}

In modeling thermal behavior of window flame, its heat source geometry needs to be

determined. Shape of the vent plane itself (a rectangle of window width $B$ at one side and length from neutral plane to upper edge $H-Z_{N}$ at the other side) is usually assumed for it.

However, unlike heat sources on the ground, heat flux profile in the vent plane is far from

uniform. The profiles of the flow velocity and the temperature rise are shown in Figs. 6 and 7,

respectively. All of the profiles take their maximum values at positions just beneath upper edges

of the windows decreasing in downward. In turn, it is reasonable to assume that a representative

point for the ejection momentum is located somewhere around the upper edge. On the other

hand, as there is not much temperature difference inside compartment in horizontal ( $x y$ ) plane,

the velocity and temperature profiles in the width-wise $(y)$ direction of the vent plane can be

assumed as uniform. Accordingly, heat source geometry of window flame is assumed as two

dimensional, in which its center is located slightly beneath the upper edge of the opening and its

profile in the width-wise $(y)$ direction is neglected.

Schematic diagram of the window flame model is shown in Fig.8. Window flame discharged 
from fire compartment initially maintains momentum in horizontal $(x)$ direction, but it gradually gains upward $(+z)$ momentum due to buoyancy. As such, trajectory of window flame is generally curved, which is distinct from that of flame located on the ground. In order to describe behavior of the curved flame appropriately, an additional two dimensional coordinate $\xi \zeta$ along the flame trajectory needs to be incorporated. First of all, the coordinate origin is defined as the location at which profiles of vent flow velocity and temperature rise take their maximum values. Curved $\xi$-axis is set along flame trajectory and $\zeta$-axis is set perpendicular to $\xi$-axis. For simplicity, interference between window flame and wall above the opening is disregarded. As window flame is nothing but the flow involving combustion of flammable gas, its phase changes along trajectory, same to the combustion phase transition of flames on the ground. The phase of window flame can be categorized into either flame region or plume region on whether it involves flammable gas combustion or not.

\subsection{Gaussian Distribution Model}

When the angle between $\xi$-axis and $x$-axis is $\theta$, points in the orthogonal coordinate are transformed into the curvilinear coordinate by the following matrix,

$$
\left(\begin{array}{l}
d \xi \\
d \zeta
\end{array}\right)=\left(\begin{array}{ll}
\frac{\partial \xi}{\partial x} & \frac{\partial \xi}{\partial z} \\
\frac{\partial \zeta}{\partial x} & \frac{\partial \zeta}{\partial z}
\end{array}\right)\left(\begin{array}{l}
d x \\
d z
\end{array}\right)=\left(\begin{array}{cc}
\cos \theta & \sin \theta \\
-\sin \theta & \cos \theta
\end{array}\right)\left(\begin{array}{l}
d x \\
d z
\end{array}\right)
$$

Assuming that window flame is the incompressible Boussinesque flow, conservation equations 
of mass, momentum (for $\xi$ - and $\zeta$ - axes), and energy appropriate for the curvilinear

coordinate are obtained by transforming the Reynolds decomposed equations for the orthogonal coordinate,

$\frac{\partial \bar{u}}{\partial \xi}+\frac{\partial \bar{w}}{\partial \zeta}=0$

$\frac{\partial \bar{u} \bar{u}}{\partial \xi}+\frac{\partial \bar{u} \bar{w}}{\partial \zeta}+\frac{\partial \overline{u^{\prime} w^{\prime}}}{\partial \zeta}=\frac{g \Delta \bar{T}}{T_{\infty}} \sin \theta$

$\bar{u} \bar{u} \frac{\partial \theta}{\partial \xi}+\frac{\partial \bar{w}^{2}}{\partial \zeta}=-\frac{1}{\rho_{\infty}} \frac{\partial \bar{p}}{\partial \zeta}+\frac{g \Delta \bar{T}}{T_{\infty}} \cos \theta$

$\frac{\partial \bar{u} \Delta \bar{T}}{\partial \xi}+\frac{\partial \bar{w} \Delta \bar{T}}{\partial \zeta}+\frac{\partial \overline{w^{\prime} \Delta T^{\prime}}}{\partial \zeta}=\frac{\overline{\dot{q}^{\prime \prime \prime}}}{\rho_{\infty} c_{p}}$

in which strain of the control volume is neglected. In these equations, $u$ and $v$ are the

velocity components, $g$ is the acceleration due to gravity, $\Delta T$ is the temperature rise relative to the ambient temperature $T_{\infty}, \rho_{\infty}$ is the ambient gas density, $C_{P}$ is the specific heat, $p$ is the pressure, and $\dot{q}^{\prime \prime \prime}$ is the heat release rate per unit volume. The superscripts $\bar{\square}$ and denote the time averaged value and fluctuation, respectively.

Now, integrating the governing Eqns.(6)-(9) in the width-wise $(\zeta$ ) direction, we obtain,

$\frac{d}{d \xi} \int_{-\infty}^{\infty} \bar{u} d \zeta+[\bar{w}]_{-\infty}^{\infty}=0$,

$\frac{d}{d \xi} \int_{-\infty}^{\infty} \bar{u}^{2} d \zeta+[\bar{u} \bar{w}]_{-\infty}^{\infty}+\left[\bar{u}^{\prime} w^{\prime}\right]_{-\infty}^{\infty}=\frac{g \sin \theta}{T_{\infty}} \int_{-\infty}^{\infty} \Delta \bar{T} d \zeta$,

$\frac{d \theta}{d \xi} \cdot \int_{-\infty}^{\infty} \bar{u}^{2} d \zeta+\left[\bar{w}^{2}\right]_{-\infty}^{\infty}=-\frac{1}{\rho_{\infty}}[\bar{p}]_{-\infty}^{\infty}+\frac{g \cos \theta}{T_{\infty}} \int_{-\infty}^{\infty} \Delta \bar{T} d \zeta$,

$\frac{d}{d \xi} \int_{-\infty}^{\infty} \bar{u} \Delta \bar{T} d \zeta+[\bar{w} \Delta \bar{T}]_{-\infty}^{\infty}+\left[\overline{w^{\prime} \Delta T^{\prime}}\right]_{-\infty}^{\infty}=\int_{-\infty}^{\infty} \frac{\overline{\dot{q}^{\prime \prime \prime}}}{\rho_{\infty} C_{P}} d \zeta$. 
Integrals involved within these equations can be removed by assuming the profiles of flow

velocity $\bar{u}$ or temperature rise $\Delta \bar{T}$ axially-symmetrical [11]. Although measured temperature data shown in Fig.5(A) supports this assumption, it may not be valid when flame attaches to the wall above the opening such as in Fig.5(B). Although adequacy of this assumption needs to be further investigated, Gaussian profiles are assumed for velocity and temperature rise regardless of trajectory configuration,

$$
\frac{\bar{u}(\xi, \zeta)}{u_{m}(\xi)}=\exp \left[-\left(\frac{\zeta}{b}\right)^{2}\right], \frac{\Delta \bar{T}(\xi, \zeta)}{\Delta T_{m}(\xi)}=\exp \left[-\beta\left(\frac{\zeta}{b}\right)^{2}\right]
$$

where $u_{m}$ is the flow velocity on the trajectory ( $\xi$-axis), $\Delta T_{m}$ is the temperature rise on the trajectory ( $\xi$-axis), $b$ is the half-width of the flow velocity, and $\beta$ is the conversion constant which reflects the difference of half-width of the flow velocity and temperature rise. In addition, assuming that the rate of air entrainment into the window flame is proportional to the central flow velocity $u_{m}[11]$,

$\lim _{\zeta \rightarrow \pm \infty} \bar{w}=-\alpha u_{m}$

where $\alpha$ is the entrainment constant.

By substituting Eqns.(14), (15) into Eqns.(10)-(13), we obtain,

$$
\frac{d}{d \xi}\left(b u_{m}\right)=\frac{2 \alpha}{\sqrt{\pi}} u_{m}
$$


$\frac{d}{d \xi}\left(b u_{m}^{2}\right)=\sqrt{\frac{2}{\beta}} g \sin \theta \cdot b \frac{\Delta T_{m}}{T_{\infty}}$

$\frac{d \theta}{d \xi} \cdot b u_{m}^{2}=\sqrt{\frac{2}{\beta}} g \cos \theta \cdot b \frac{\Delta T_{m}}{T_{\infty}}$

$\frac{d}{d \xi}\left(b u_{m} \frac{\Delta T_{m}}{T_{\infty}}\right)=\sqrt{\frac{1+\beta}{\pi}} \int_{-\infty}^{\infty} \frac{\overline{\dot{q}^{\prime \prime \prime}}}{\rho_{\infty} C_{P} T_{\infty}} d \zeta$

in which the terms $\bar{u} \bar{w}, \overline{u^{\prime} w^{\prime}}, \bar{w}^{2}, \bar{p}, \bar{w} \Delta \bar{T}, \overline{w^{\prime} \Delta T^{\prime}}$ were all assumed to be 0 when $\varsigma \rightarrow \pm \infty$, i.e., at ambient environment. In the derived equations, the heat release rate term $\bar{q}^{\prime \prime \prime}$ in Eqn.(19) will take different forms depending on its belonging region: the flame region; or the plume region. As to the flame region, Assuming that the heat release rate is controlled by the rate of air entrainment,

$\int_{-\infty}^{\infty} \bar{q}^{\prime \prime \prime} d \zeta=\left(1-\chi_{R}\right) \frac{\Delta H_{F}}{n \gamma} \cdot 2 \rho_{\infty} \alpha u_{m}$

where $\chi_{R}$ is the rate of radiation heat loss, $\Delta H_{F}$ is the heat of combustion of the flammable gas, $\gamma$ is the theoretical fuel/air ratio, and $n$ is the correction factor which represents the difference of the fuel/air ratio between theoretical and practical. Substituting Eqn.(20) into Eqn.(19), the conservation equation of energy for the flame region is derived as,

$$
\frac{d}{d \xi}\left(b u_{m} \frac{\Delta T_{m}}{T_{\infty}}\right)=\left(1-\chi_{R}\right) \sqrt{\frac{1+\beta}{\pi}} \frac{\Delta H_{F}}{n \gamma \cdot c_{P} T_{\infty}} \cdot 2 \alpha u_{m}
$$

On the other hand, in the plume region, where no combustion is taken place, the heat release rate $\overline{\dot{q}^{\prime \prime \prime}}$ becomes zero, 
$\rho_{\infty} C_{P} T_{\infty} \sqrt{\frac{\pi}{1+\beta}} \frac{d}{d \xi}\left(b u_{m} \frac{\Delta T_{m}}{T_{\infty}}\right)=0$

This equation shows that total amount of energy maintained inside the plume is unchanged

along $\xi$-axis. Thus, Eqn.(22) can be integrated to give,

$b u_{m} \frac{\Delta T_{m}}{T_{\infty}}=\sqrt{\frac{1+\beta}{\pi}} \frac{\left(1-\chi_{R}\right) \dot{Q}^{\prime}}{\rho_{\infty} c_{P} T_{\infty}}$

where $\left(1-\chi_{R}\right) \dot{Q}^{\prime}$ is the overall heat release rate of the window flame in which the portion of

radiation heat loss is excluded.

\subsection{Scaling Relations}

The obtained conservation equations need to be normalized in order to analyze dimensional

relations between the involved parameters. With the normalizing parameters for length $D$, flow

velocity $U$, and temperature $T$, dimensionless parameters are defined as,

$\xi^{*} \equiv \frac{\xi}{D}, \quad B^{*} \equiv \frac{b}{D}, \quad U^{*} \equiv \frac{u_{m}}{U}, \quad T^{*} \equiv \frac{\Delta T_{m}}{T}$

where the superscript $\square^{*}$ denotes that the parameter is dimensionless. By substituting these

parameters into Eqns.(16), (17), (18), (21), (23), we obtain the conservation equations in

dimensionless form, respectively,

mass: $\frac{d}{d \xi^{*}}\left(B^{*} U^{*}\right)=\frac{2 \alpha}{\sqrt{\pi}} U^{*}$

momentum $(\xi): \frac{d}{d \xi^{*}}\left(B^{*} U^{* 2}\right)=\frac{g D T}{U^{2} T_{\infty}} \sqrt{\frac{2}{\beta}} \sin \theta \cdot B^{*} T^{*}$ 
momentum $(\zeta): \frac{d \theta}{d \xi^{*}} \cdot U^{* 2}=\sqrt{\frac{2}{\beta}}\left(\frac{\sqrt{g D}}{U}\right)^{2} \cos \theta \cdot T^{*}$

energy (flame region): $\frac{d}{d \xi^{*}}\left(B^{*} U^{*} T^{*}\right)=2 \alpha\left(1-\chi_{R}\right) \sqrt{\frac{1+\beta}{\pi}}\left(\frac{\Delta H_{F}}{n \gamma \cdot c_{P} T_{\infty}}\right) \cdot U^{*}$

energy (plume region): $B^{*} U^{*} T^{*}=\left(1-\chi_{R}\right) \sqrt{\frac{1+\beta}{\pi}} \frac{\dot{Q}^{\prime}}{\rho_{\infty} C_{p} D U T}$

Before implementing dimensional analysis, specific values for the normalizing parameters $D$,

$U$ and $T$ need to be determined. It is empirically known that change in the width does not

change as much as the length along trajectory [12]. So we adopt the initial width of window

flame, i.e., the width of the venting plane, for the normalizing parameter $D$,

$D=H-Z_{N}$

Whereas for the rest of the normalizing parameters $U$ and $T$, terms in Eqns.(26) and (29) are equated to one, so that it becomes dimensionless [13],

$\frac{g D T}{U^{2} T_{\infty}}=1, \frac{\dot{Q}^{\prime}}{\rho_{\infty} C_{p} D U T}=1$

which allows us to determine $U$ and $T$ as,

$U=g^{1 / 2}\left(\frac{\dot{Q}^{\prime}}{\rho_{\infty} C_{P} T_{\infty} g^{1 / 2}}\right)^{1 / 3}, T=\left(\frac{\dot{Q}^{\prime}}{\rho_{\infty} C_{P} T_{\infty} g^{1 / 2}\left(H-Z_{N}\right)^{3 / 2}}\right)^{2 / 3} T_{\infty}$

To summarize, the dimensionless parameters in Eqn.(24) can be expressed as follows,

$$
\xi^{*} \equiv \frac{\xi}{H-Z_{N}}, \quad B^{*} \equiv \frac{b}{H-Z_{N}}, \quad U^{*} \equiv \frac{u_{m} \cdot Q^{\prime *-1 / 3}}{\sqrt{g\left(H-Z_{N}\right)}}, \quad T^{*} \equiv \frac{\Delta T_{m} \cdot Q^{\prime *-2 / 3}}{T_{\infty}}
$$


where $Q^{\prime *}$ is another dimensionless parameter which is defined as follows,

$$
Q^{\prime *} \equiv \frac{\dot{Q}^{\prime}}{\rho_{\infty} C_{P} T_{\infty} g^{1 / 2}\left(H-Z_{N}\right)^{3 / 2}}
$$

As the behavior of window flame may change as getting apart from the venting plane, the characteristic parameters $B^{*}, U^{*}$ and $T^{*}$ are expected to be some power functions of the dimensionless parameter $\xi^{*}$, which represents the distance along trajectory, i.e.,

$$
B^{*} \propto\left(\xi^{*}\right)^{l}, U^{*} \propto\left(\xi^{*}\right)^{m}, T^{*} \propto\left(\xi^{*}\right)^{n}
$$

where $l, m$ and $n$ are the real numbers. Substituting them into the dimensionless

Eqns.(25)-(29), we obtain $\xi^{*}$ on both sides of the equations. As multipliers of $\xi^{*}$ in the both sides should coincide, real numbers $l, m$ and $n$ are taken into simultaneous equations. By solving these equations, scale relations are obtained for the flame region and plume region, respectively,

flame region $\left\{\begin{array}{l}B^{*} \propto \xi^{*} \\ U^{*} \propto \xi^{* 1 / 2}, \\ T^{*}=\text { const. }\end{array} \quad\right.$ plume region $\left\{\begin{array}{l}B^{*} \propto \xi^{*} \\ U^{*}=\text { const. } \\ T^{*} \propto \xi^{*-1}\end{array}\right.$ 


\section{VERIFICATION OF THE SCALE MODEL}

\subsection{Temperature Rise along the Trajectory}

Transforming the derived scaling expression for the temperature rise in Eqn.(36) into a partially finite-dimensional form,

$\frac{\Delta T_{m}}{T_{\infty}} \propto \begin{cases}Q^{\prime \prime 2 / 3} & \text { (flame region) } \\ Q^{\prime \prime 2 / 3}\left(\frac{\xi}{H-Z_{N}}\right)^{-1} & \text { (plume region) }\end{cases}$

Correlating experimental data with the above expression, we obtain Fig.9. Plots of the

dimensionless temperature rises take the maximum and constant values near the vent plane, and after that, it decrease at a rate almost proportional to $\xi^{-1}$. Although there is some variation, the results show pertinent consistency with the estimated expression in Eqn.(37). Expressions for the temperature rise prediction of window flames can be obtained by drawing regression lines to these plots. However, the lines should envelop the entire plots in order to avoid the underestimation of window flame hazard.

$$
\frac{\Delta T_{m}}{T_{\infty}}= \begin{cases}2.0 Q^{\prime * 2 / 3} & \text { (flame region : } \left.\frac{\xi}{H-Z_{N}} \leq 0.64\right) \\ 1.6 Q^{, * 2 / 3}\left(\frac{\xi}{H-Z_{N}}\right)^{-1 / 2} & \text { (intermittent region : } \left.0.64<\frac{\xi}{H-Z_{N}} \leq 2.44\right) \\ 2.5 Q^{\prime 22 / 3}\left(\frac{\xi}{H-Z_{N}}\right)^{-1} & \text { (plume region : } \left.2.44<\frac{\xi}{H-Z_{N}}\right)\end{cases}
$$

where the intermediate region between the flame region and plume region is named as the 
intermittent region. The power for the intermittent region was adjusted to $-1 / 2$, which is a mean value of 0 for the flame region and -1 for the plume region. Note again that $\xi$ is distance along trajectory and trajectory is generally curved due to buoyancy. As a result, calculation of $\Delta T_{m}$ may become complex as $\xi$ is not always easy to evaluate. As an alternative, we can substitute $\xi$ with the relevant vertical height $z$. As the trajectory is curved and $\xi$ is always larger than $z$, predicted result with $z$ exclusively gives higher $\Delta T_{m}$ than that with $\xi$. In other words the prediction of $\Delta T_{m}$ always involves error which somewhat overestimate the window flame hazard.

In deriving the scale model, we approximated window flame behavior by two dimensional flow. However in actual conditions, it is expected that window flame acquires some characteristics typical to three dimensional flow as getting apart from the vent plane. This is because the flow starts entraining ambient air from all directions while ascending. In such a case, hot current of window flame is even more diluted, and the rate of temperature decrease should become faster, i.e., the power function of $\quad \xi$ should become less than -1 . However, as far as the results in Fig.9 shows, no significant sign of such transition was observed. One of the possible reasons for this is due to the restriction of air entrainment as the flame ascended along the wall.

\subsection{Half-width of the Gaussian Model}

Following the derived relations in Eqn.(36), the half-width of the flow velocity distribution $b$ 
enlarges along the trajectory at a rate proportional to $\xi$, either in the flame region or plume region. However, we were unable to measure $b$ for the velocity due to a constraint of the experimental apparatus. As the half-width of the temperature rise distribution $b_{T}$ is also proportional to both $b$ and $\xi$ in the model, we alternatively verify the model with the following relation,

$b_{T} \propto b \propto \xi$

The results for the open side of the trajectory is shown in Fig.10(A), and those for the wall side is shown in Fig.10(B). As thermocouples located close to the opening receive substantial radiation heat from compartment gas, data acquired at thermocouples higher than $0.2 \mathrm{~m}$ from the upper edge of the opening were analyzed. Consequently, most of the plots in Figs.10(A) and (B) are the ones in the plume region. Also note that the number of plots in Fig.10(B) for the wall side is less than that of Fig.10(A) for the open side, $b_{T}$ was not distinguishable when window flames attached to the wall.

Although there is some variations in the plots due to the scarce alignment of thermocouples, there is a fairly clear correlation of linearity between $\quad b_{T}$ and $\xi$. Drawing regressive lines to the plots in both Figs.10(A) and (B), we obtain,

open side: $\quad b_{T}=0.04+0.20 \xi \quad(\mathrm{r}=0.82)$ 
wall side: $\quad b_{T}=0.06+0.20 \xi \quad(\mathrm{r}=0.73)$

These are shown as broken lines. As evident from the above Eqns.(40) and (41), the two

regression lines almost coincide with each other. In other words, the temperature distributions

were almost axially symmetrical for the window flames not attaching to the wall. Solid line

drawn in Figs.10(A) and (B) is the regression line for the overall plots, which is given as,

$b_{T}=0.05+0.20 \xi \quad(\mathrm{r}=0.80)$.

These results show that the proportion of the half-width of temperature rise distribution to the

length along trajectory is about $1 / 5$. To compare with the relevant values in the existing

experiments, Sugawa et al. reported 1/5 [4] and Ohmiya et al. reported 1/3-1/4 [9], which are compatible with our result. Whereas for the vertically rising fire plume with the heat source placed on the floor, the relevant value of 1/10 is reported by Quintiere et al. [12].

\subsection{Comparison with the Experimental Data}

To further verify our proposed model, temperature rises predicted by the model were compared with the experimental data. The results are shown in Figs.11-15. The comparison involves the results for 5 different opening dimensions and 2 different air supply rates. Predictions by the model proposed by Yokoi are also traced in the figures. Yokoi's model takes the following form $[1,14]$ 
$\frac{Z}{r_{0}}= \begin{cases}1.05 \Theta^{-1} & (\Theta \leq 0.35) \\ 0.00193 \Theta^{-7} & (0.35<\Theta)\end{cases}$

where $\Theta$ is the scaling dimensionless parameter based upon the point heat source assumption

[11]. The parameter $\Theta$ is defined as,

$\Theta \equiv \frac{\Delta T_{m} \cdot r_{0}^{5 / 3}}{\left(\dot{Q}^{2} T_{\infty} / c_{P}^{2} \rho^{2} g\right)^{1 / 3}}$

where, $r_{0}\left(=\sqrt{B\left(H-Z_{N}\right) / \pi}\right)$ is the equivalent radius of the opening.

As the model parameters were adjusted not to underestimate the window flame hazard in the

both models, predicted temperature rises were somewhat higher than the experimental values in

general. However, the agreements of the present model were slightly better than those of

Yokoi's model. Nevertheless, we should take into account that experimental data used for this

temperature rise comparison are those used for determining model parameters in the present

model. One of the important differences in the two models is the scaling dimensionless

parameter: the one for the present model is $Q^{\prime *}$ for the two dimensional flow downstream of a line heat source; and the one for Yokoi's model is $\Theta$ for the three dimensional flow downstream of a point heat source. The difference attributed to these model parameters as well as their multipliers can be observed in Figs.11-15, in that reduction rates in the predicted temperature rise were larger in those of Yokoi's model than the present model. 


\section{CONCLUSIONS}

In this study, a series of reduced scale experiment on window flame ejection was carried out.

Fresh air was forcibly introduced into the compartment with a mechanical fan, in order to

investigate the effect of wind affected pressure rise inside fire compartment on behavior of

window flame. Concurrently, a characteristic dimensionless parameter for the thermal behavior

of window flame $Q^{\prime *}$ was derived from the governing equations. The obtained experimental

results were generalized with $Q^{\prime *}$, providing new equations for predicting temperature rise

along trajectory $\Delta T_{m}$ and half-width of temperature rise distribution $b_{T}$. Predicted results of

the model were compared with the experimental data and reasonable agreements were obtained.

Future works are listed as follows:

1) Dimension of the compartment used in the present experiment was about $1 / 4-1 / 3$ of the

full scale. As the model proposed in this study is a scale model, comparison with other

experimental data at different scales will be beneficial in strengthening its validity.

2) As velocity measurement outside the compartment was not conducted in this study, ones of

the derived model parameters are still not determined. They need to be correlated with

additional measurement.

3) A model for configuration of window flame trajectory is needed as the present model

predicts temperature rise along the trajectory. This model will be valid when estimating the 
rate of heat transfer to the adjacent objects somewhat apart from the window flame.

4) The effect of wind on window flame behavior was investigated in terms of pressure rise inside the fire compartment. However, temperature rise and geometry of window flame may change when it is blown directly by the wind. They are another important aspect which needs further consideration.

\section{REFERENCES}

1. Yokoi S. Study on the prevention of fire-spread caused by hot upward current. Report of the Building Research Institute, No.34. 1960.

2. Thomas PH, Law M. The projection of flames from burning buildings. Fire Research Note, No.921. 1972

3. Oleszkiewicz I. Fire exposure to exterior walls and flame spread on combustible cladding.

Fire Technology 1990; 26(4): 357-375.

4. Sugawa O, Momita D, Takahashi W. Flow behavior of ejected fire flame/plume from an opening effected by external side wind. Proc. 5th Symp., pp.249-260. International Association for Fire Safety Science, 1997.

5. Poreh M, Morgan HP, Marshall NR, Harrison R. Entrainment by two-dimensional spill plume.

Fire Safety J 1998; 30: 1-19. 
6. Thomas PH, Morgan HP, Marshall N. The spill smoke in smoke control design. Fire Safety J 1998; 30: 21-46.

7. Klopovic S, Turan OF. Flames venting externally during full-scale flashover fires: two sample ventilation cases. Fire Safety J 1998; 31: 117-142.

8. Yamaguchi J, Iwai Y, Tanaka T, Harada K, Ohmiya Y, Wakamatsu T. Applicability of nondimensional temperature for scaling the temperatures of window jet plume. $J$ Architectural Planning and Environmental Engineering 1998; 513: 1-7 (in Japanese).

9. Ohmiya Y, Hori Y. Properties of external flame taking into consideration excess fuel gas ejected from fire compartment. J Architectural Planning and Environmental Engineering, 2001; 546: 1-8 (in Japanese).

10. Yamada T, Takanashi K, Yanai E, Suzuki T, Sekizawa A. An experimental study of ejected flames and combustion efficiency. Proc. $7^{\text {th }}$ Int. Symp., pp.903-914. International Association for Fire Safety Science, 2002.

11. Morton BR, Taylor GI, TurnerJS. Turbulent gravitational convection from maintained and instantaneous sources. Proc. Royal Society 1956; 234A: 1-23.

12. Quintiere JG, Grove BS. A unified analysis for fire plumes. Proc. $27^{\text {th }}$ Symp. (Int.) Comb., pp.2757-2766, The Combustion Institute, 1998.

13. Quintiere JG. Scaling applications in fire research. Fire Safety J 1989; 15: 3-29. 
14. Recommendation on fire resistant design of steel structures. Architectural Institute of Japan

1999 (in Japanese).

\section{NOMENCLATURE}

\section{Alphabets}

A : Area of the opening $\left(\mathrm{m}^{2}\right)$

$b \quad$ :Half width of the flow velocity (m)

$b_{T} \quad$ :Half width of the flow temperature rise (m)

$B \quad$ :Horizontal width of the opening (m)

$B^{*} \quad$ :Dimensionless half width (-)

$c_{P} \quad:$ Heat capacity of the flow $(\mathrm{kJ} /(\mathrm{kgK}))$

D :Characteristic normalizing length (m)

$g \quad$ :Acceleration due to gravity $\left(\mathrm{m} / \mathrm{s}^{2}\right)$

$H \quad$ :Height of the opening (m)

$\dot{m}_{F} \quad$ :Mass loss rate of the fuel $(\mathrm{kg} / \mathrm{s})$

$\dot{m}_{0} \quad$ :Air supply rate $(\mathrm{kg} / \mathrm{s})$

$\dot{q}^{\prime \prime \prime} \quad$ :Heat release rate of the window flame per unit volume $\left(\mathrm{kW} / \mathrm{m}^{3}\right)$

$\dot{Q} \quad$ :Apparent heat release rate of the window flame $(\mathrm{kW})$ 
$\dot{Q}^{\prime} \quad$ : Apparent heat release rate of the window flame per unit length $(\mathrm{kW} / \mathrm{m})$

$Q^{\prime *} \quad$ :Dimensionless heat release rate (-)

$r_{0} \quad$ :Equivalent radius of the opening (m)

$T \quad$ :Characteristic normalizing temperature (K)

$\Delta T \quad$ :Flow temperature rise (K)

$\Delta T_{m} \quad$ :Flow temperature rise along trajectory (K)

$T_{\infty} \quad$ :Ambient gas temperature (K)

$T^{*} \quad$ :Dimensionless temperature rise (-)

$u \quad$ :Flow velocity ( $\xi$-component) $(\mathrm{m} / \mathrm{s})$

$u_{m} \quad$ :Flow velocity along trajectory $(\mathrm{m} / \mathrm{s})$

$u_{0} \quad$ :Maximum flow velocity at the opening $(\mathrm{m} / \mathrm{s})$

$U \quad$ :Characteristic normalizing velocity $(\mathrm{m} / \mathrm{s})$

$U^{*} \quad$ :Dimensionless velocity (-)

$v \quad$ :Flow velocity ( $\zeta$-component) $(\mathrm{m} / \mathrm{s})$

$Z_{N} \quad$ :Height of the neutral plane (m)

\section{Greeks}

$\zeta \quad$ :Separation from the trajectory (m)

$\theta \quad$ :Angle between $x$-axis and $\xi$-axis (rad) 
$\Theta \quad$ :Dimensionless heat release rate (-)

$\xi \quad$ :Distance along trajectory (m)

$\xi^{*} \quad$ :Dimensionless distance (-)

$\pi \quad$ :Circumference ratio (-)

$\rho_{\infty} \quad$ :Ambient gas density $\left(\mathrm{kg} / \mathrm{m}^{3}\right)$

\section{Others}

$\bar{\square} \quad$ :Mean

$\square^{*} \quad$ :Dimensionless number

:Per unit time

$\square^{\prime} \quad$ :Per unit length, or fluctuation

$\square " \quad$ :Per unit area

$\square^{\prime \prime \prime} \quad$ :Per unit volume 


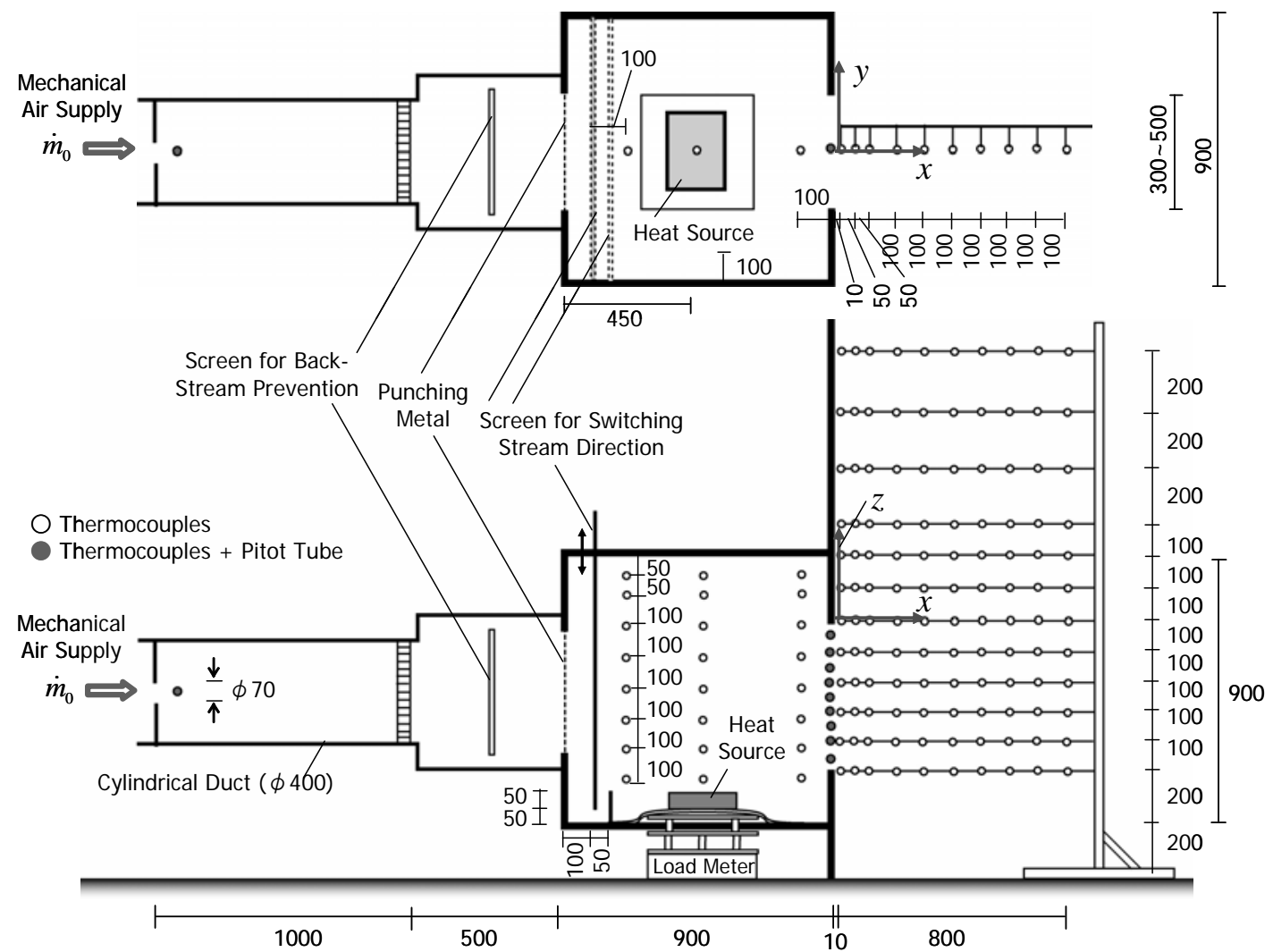

Fig. 1. Horizontal projection and cross-section of the compartment. Measuring points for flow temperature and velocity are also indicated. Units are in millimeters (mm). 


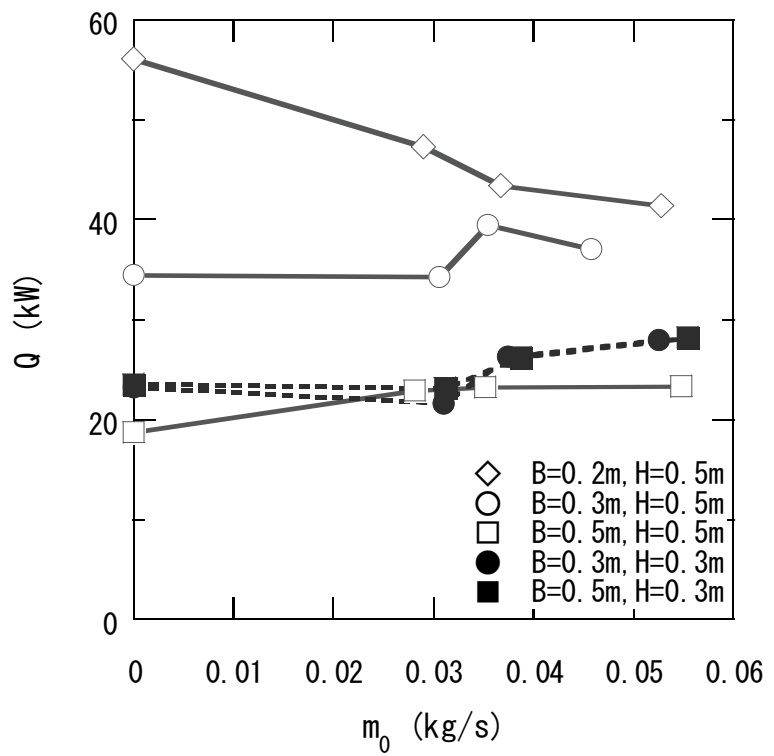

Fig. 2. Mass supply rate by the fan and the virtual heat release rate of window flame. 


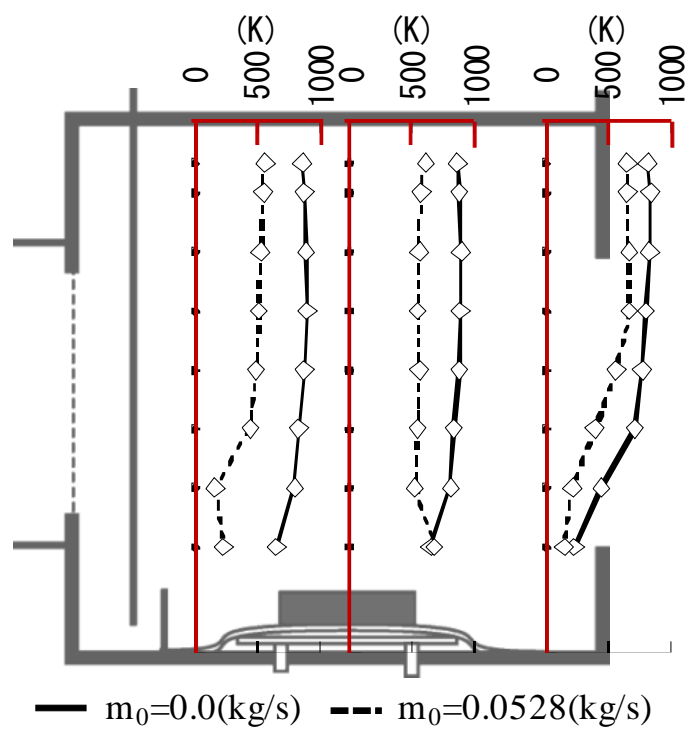

(A) $B=0.2 \mathrm{~m}, \quad H=0.5 \mathrm{~m}$

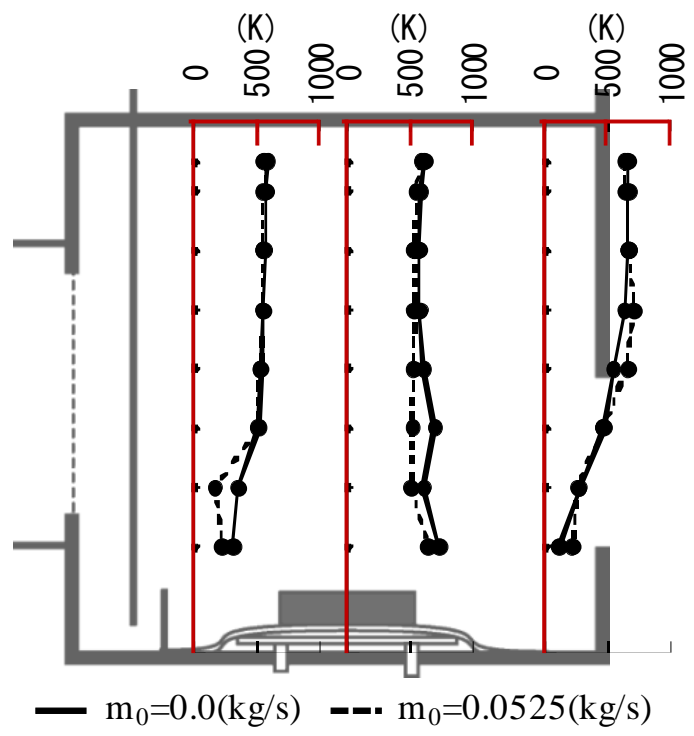

(B) $B=0.3 \mathrm{~m}, \quad H=0.3 \mathrm{~m}$

Fig.3. Mass supply rate and compartment gas temperatures. Lines of the thermocouple locations represent the zero axes for the temperature plots. 


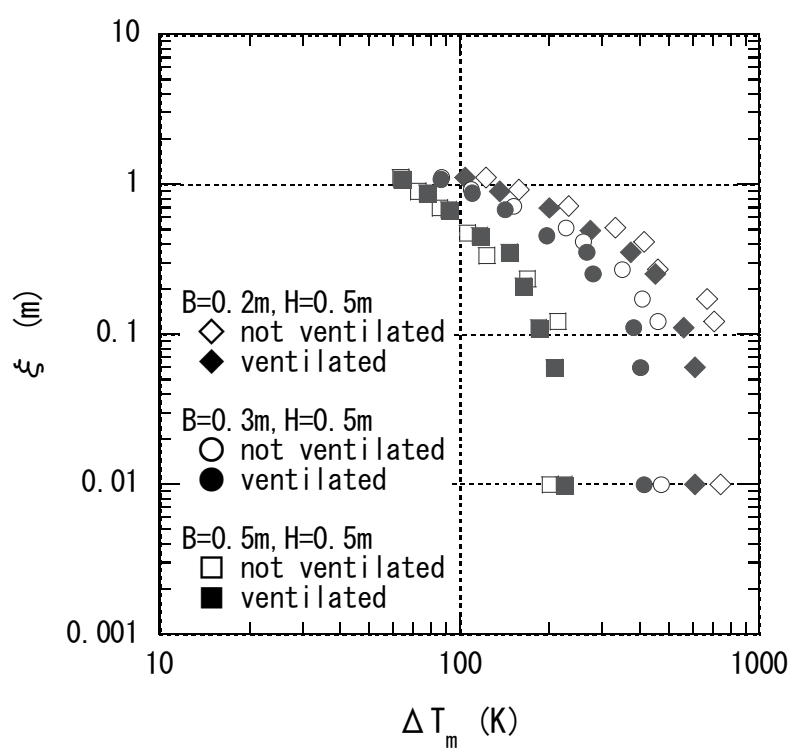

Fig. 4. Effect of the mechanical air supply on the window flame temperature along trajectory. 


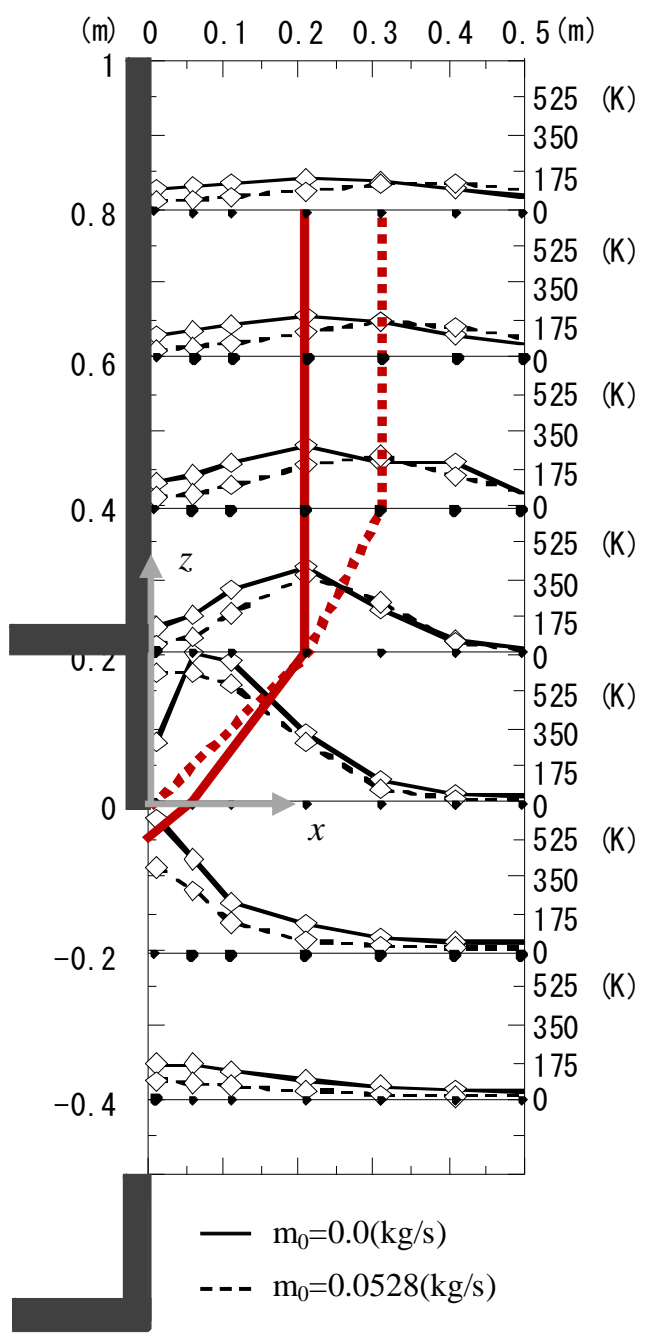

(A) $B=0.2 \mathrm{~m}, \quad H=0.5 \mathrm{~m}$

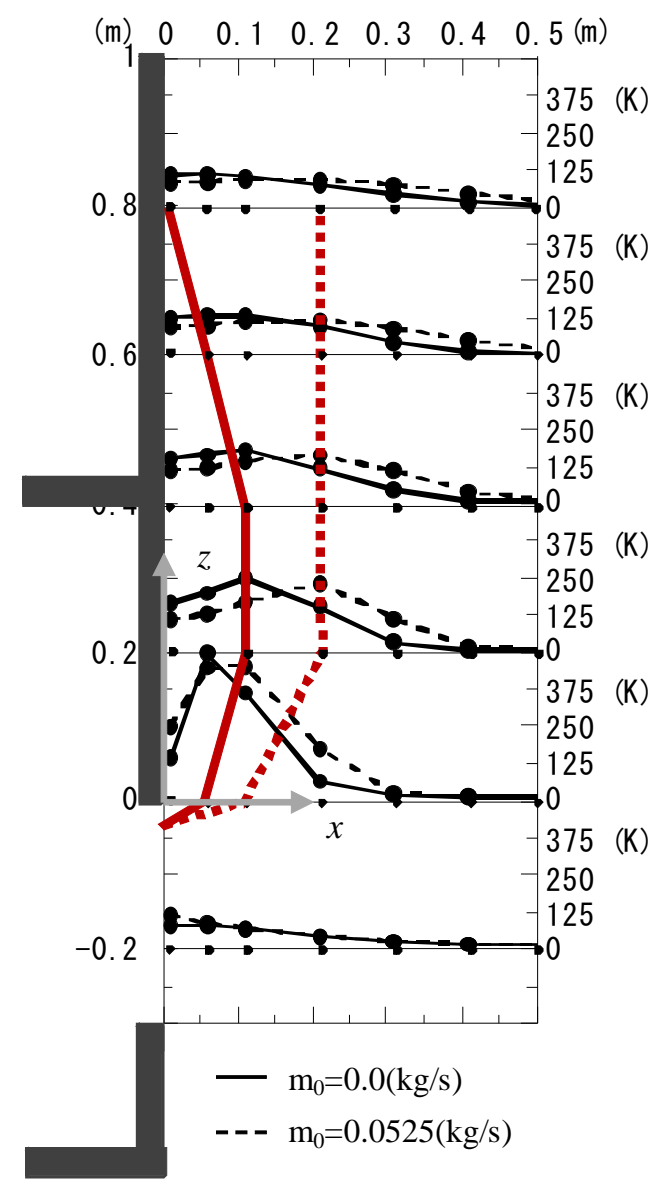

(B) $B=0.3 \mathrm{~m}, \quad H=0.3 \mathrm{~m}$

Fig. 5. Examples of the temperature rise and trajectory at different inflow rate by the fan. Lines of the thermocouple locations represent the zero axes for the temperature plots. 


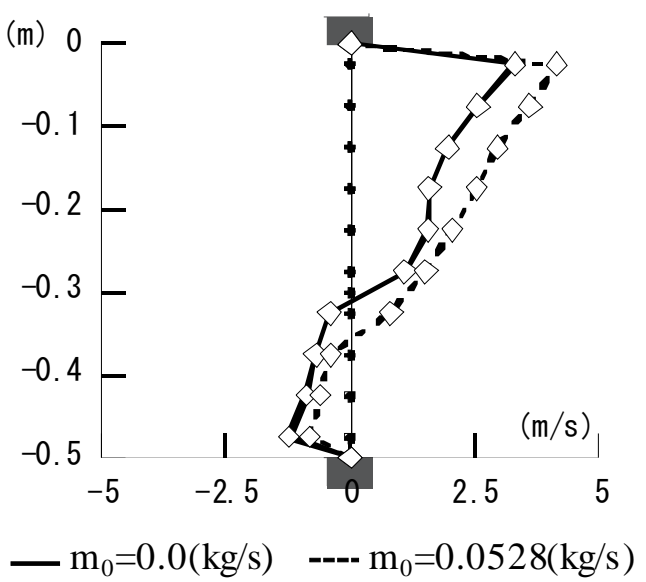

(A) $B=0.2 \mathrm{~m}, \quad H=0.5 \mathrm{~m}$

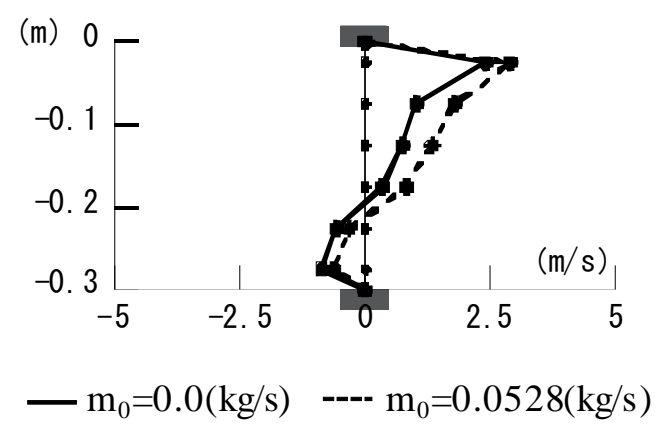

(B) $B=0.3 \mathrm{~m}, \quad H=0.3 \mathrm{~m}$

Fig.6. Flow velocity profiles at the window plane $(y=0)$. 


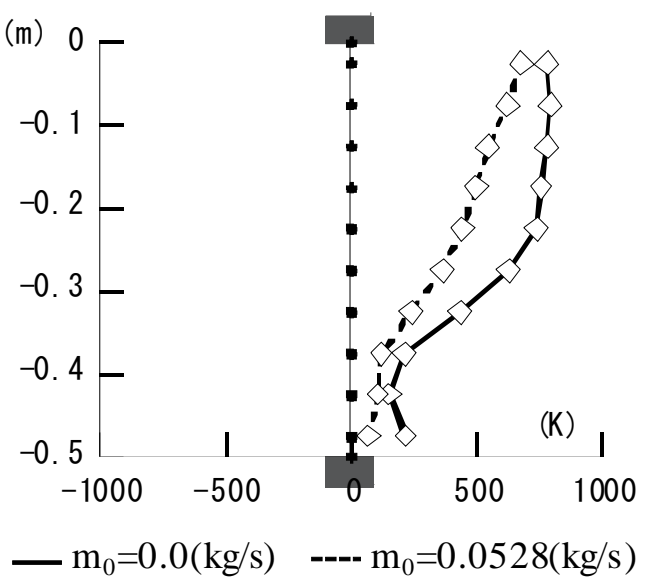

(A) $B=0.2 \mathrm{~m}, \quad H=0.5 \mathrm{~m}$

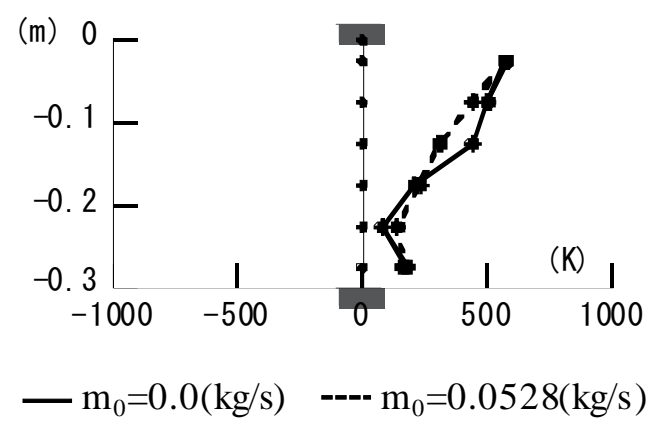

(B) $B=0.3 \mathrm{~m}, \quad H=0.3 \mathrm{~m}$

Fig.7. Temperature profile at the window plane $(y=0)$. 


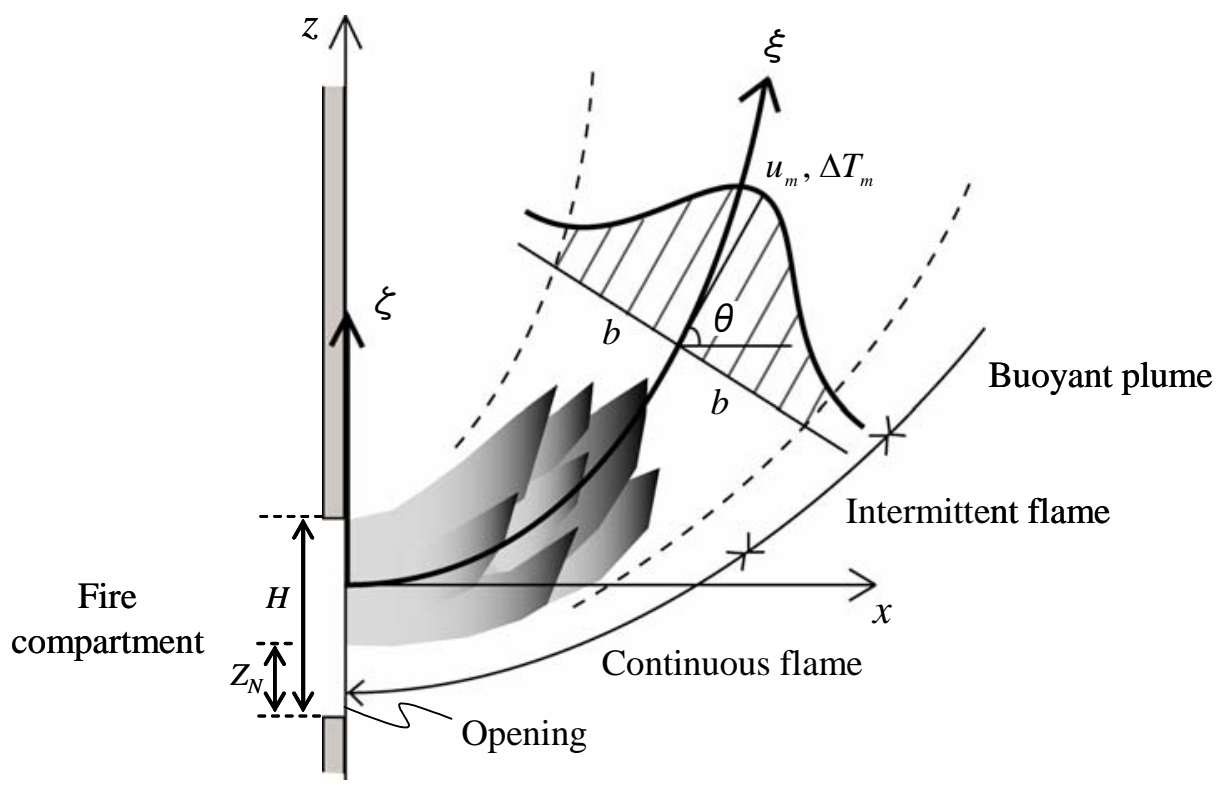

Fig. 8. Schematic diagram of the window flame model. 


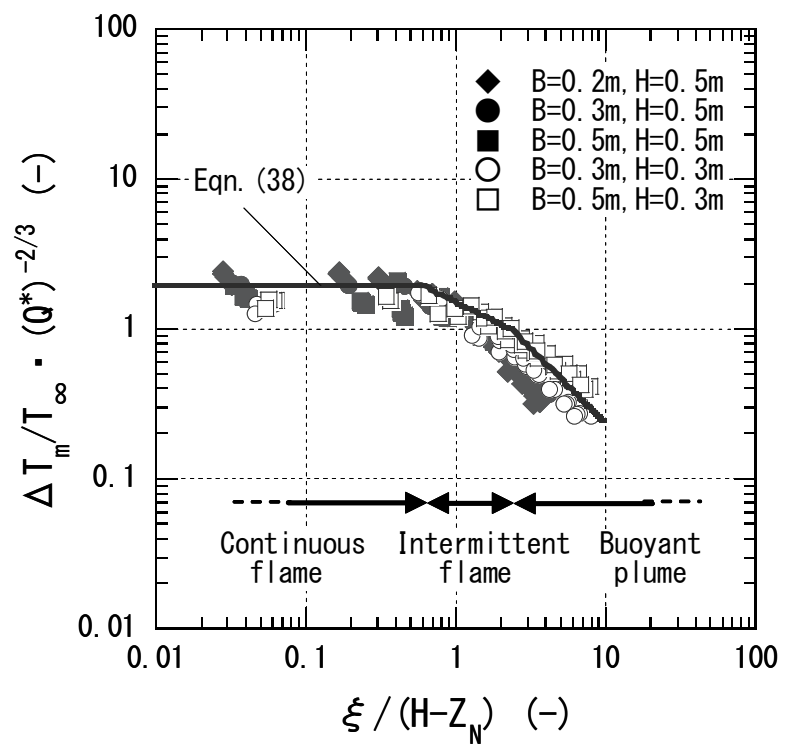

Fig. 9. Generalized temperature rise along window flame trajectory. 


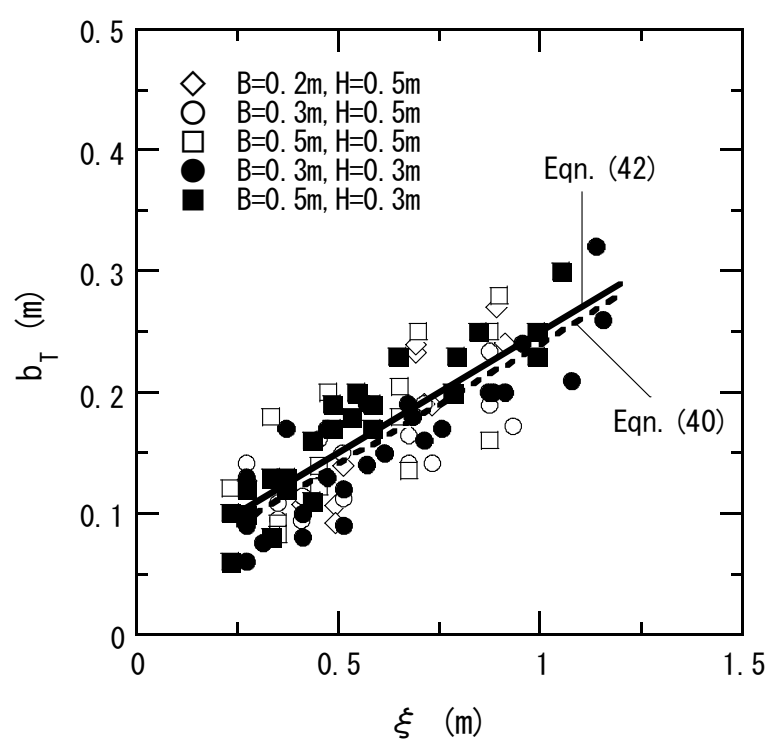

(A) Open side of the window flame

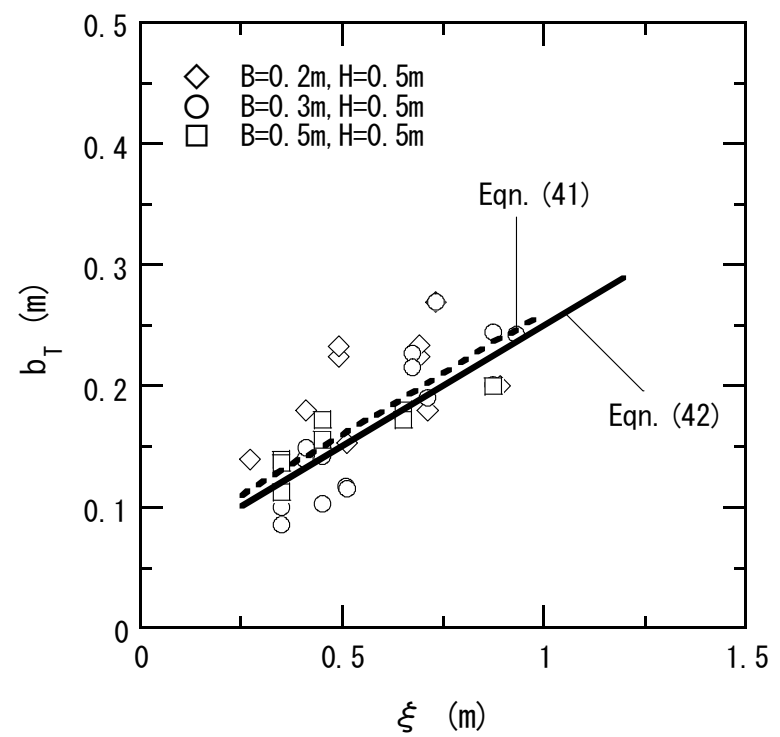

(B) Wall side of the window flame

Fig.10. Half width of the temperature rise along window flame trajectory. 


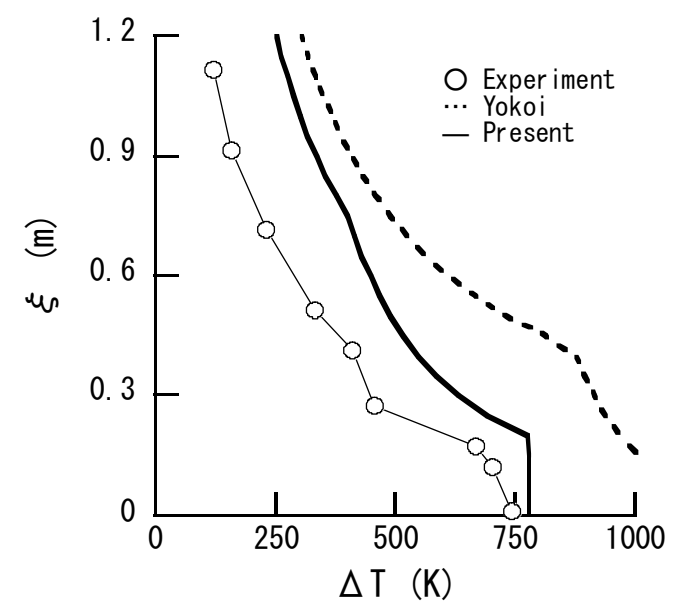

(A) $B=0.2 \mathrm{~m}, \quad H=0.5 \mathrm{~m}, \quad \dot{m}_{0}=0.0 \mathrm{~kg} / \mathrm{s}$

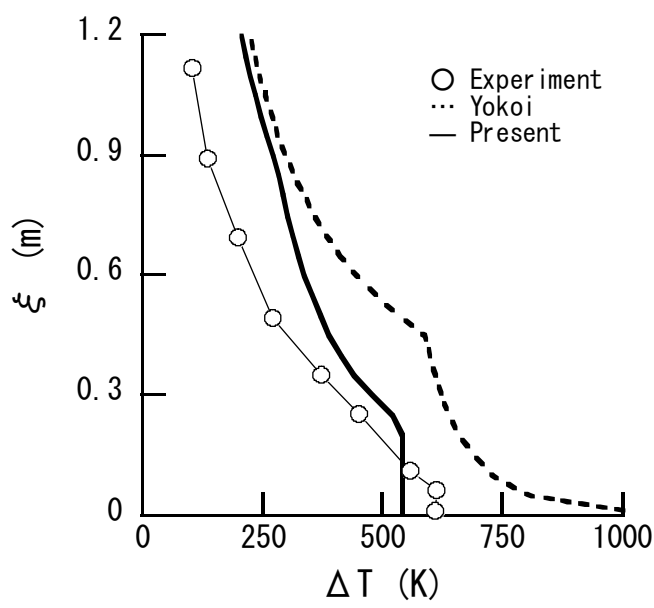

(B) $B=0.2 \mathrm{~m}, \quad H=0.5 \mathrm{~m}, \quad \dot{m}_{0}=0.0528 \mathrm{~kg} / \mathrm{s}$

Fig.11. Comparison of the temperature rise along trajectory ( $B=0.2 \mathrm{~m}, \quad H=0.5 \mathrm{~m})$ 


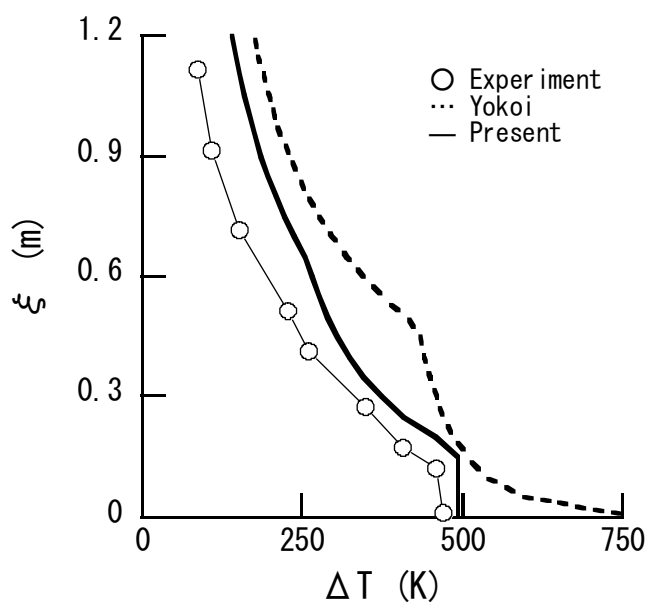

(A) $B=0.3 \mathrm{~m}, \quad H=0.5 \mathrm{~m}, \quad \dot{m}_{0}=0.0 \mathrm{~kg} / \mathrm{s}$

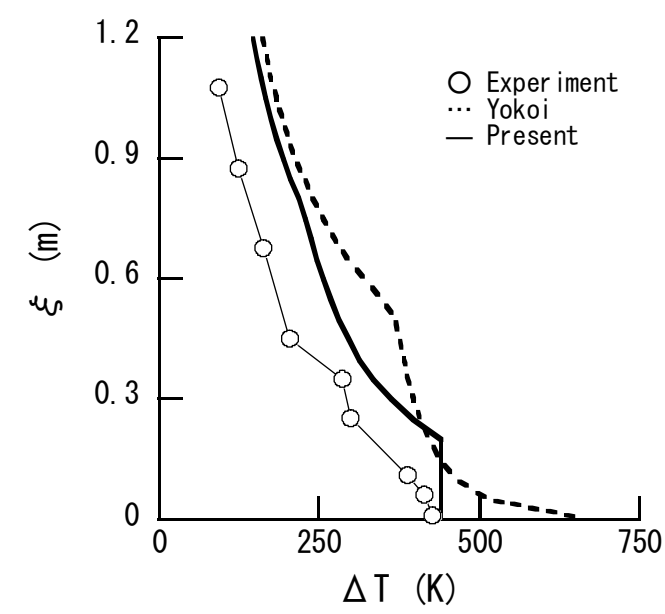

(B) $B=0.3 \mathrm{~m}, \quad H=0.5 \mathrm{~m}, \quad \dot{m}_{0}=0.0458 \mathrm{~kg} / \mathrm{s}$

Fig.12. Comparison of the temperature rise along trajectory $(B=0.3 \mathrm{~m}, \quad H=0.5 \mathrm{~m})$ 


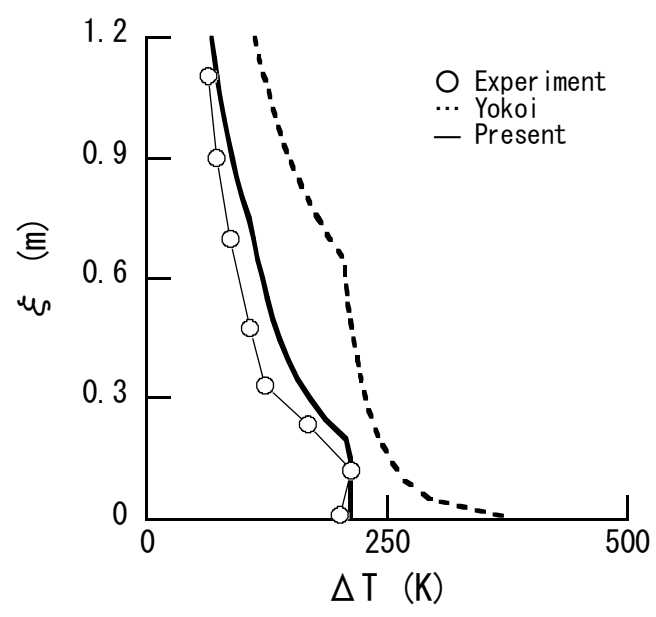

(A) $B=0.5 \mathrm{~m}, \quad H=0.5 \mathrm{~m}, \quad \dot{m}_{0}=0.0 \mathrm{~kg} / \mathrm{s}$

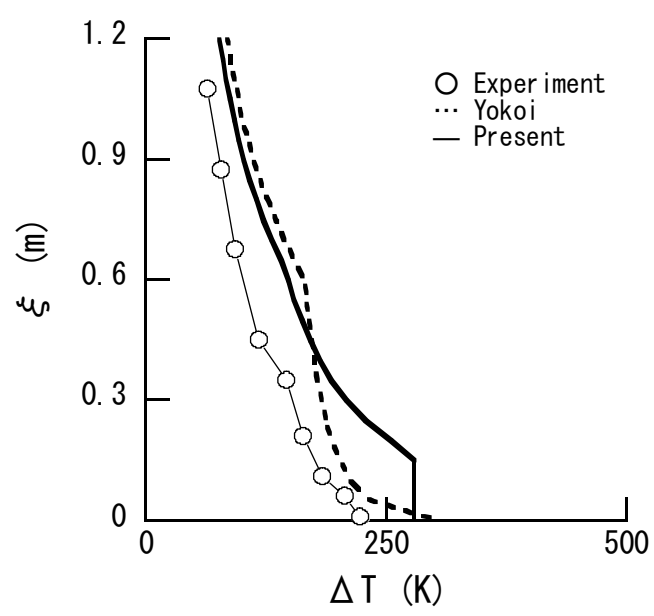

(B) $B=0.5 \mathrm{~m}, H=0.5 \mathrm{~m}, \quad \dot{m}_{0}=0.0548 \mathrm{~kg} / \mathrm{s}$

Fig.13. Comparison of the temperature rise along trajectory ( $B=0.5 \mathrm{~m}, H=0.5 \mathrm{~m}$ ) 


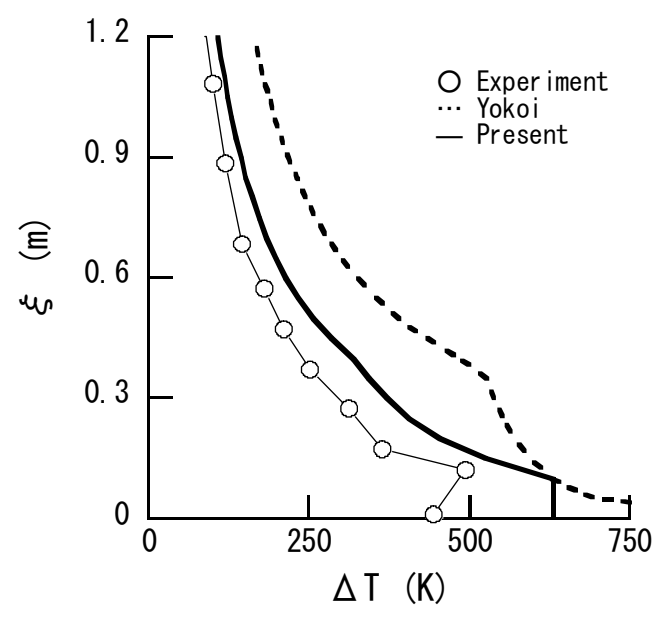

(A) $B=0.3 \mathrm{~m}, \quad H=0.3 \mathrm{~m}, \quad \dot{m}_{0}=0.0 \mathrm{~kg} / \mathrm{s}$

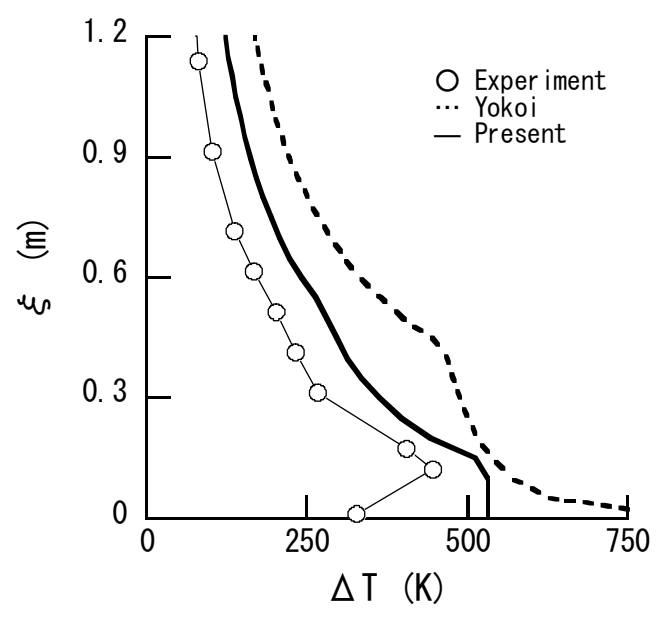

(B) $B=0.3 \mathrm{~m}, \quad H=0.3 \mathrm{~m}, \quad \dot{m}_{0}=0.0525 \mathrm{~kg} / \mathrm{s}$

Fig.14. Comparison of the temperature rise along trajectory ( $B=0.3 \mathrm{~m}, \quad H=0.3 \mathrm{~m}$ ) 


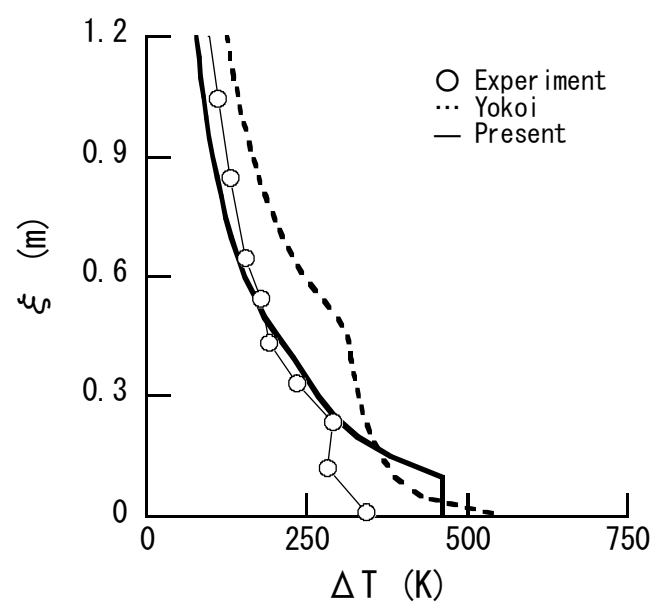

(A) $B=0.5 \mathrm{~m}, \quad H=0.3 \mathrm{~m}, \quad \dot{m}_{0}=0.0 \mathrm{~kg} / \mathrm{s}$

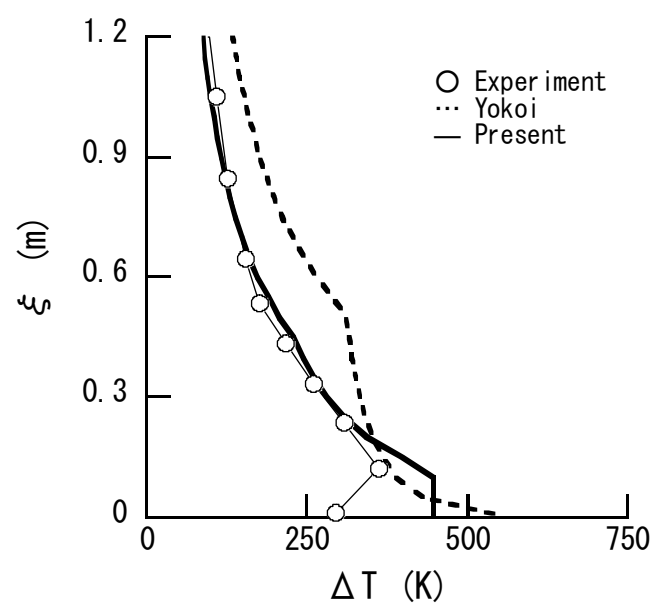

(B) $B=0.5 \mathrm{~m}, \quad H=0.3 \mathrm{~m}, \quad \dot{m}_{0}=0.0554 \mathrm{~kg} / \mathrm{s}$

Fig.15. Comparison of the temperature rise along trajectory $(B=0.5 \mathrm{~m}, \quad H=0.3 \mathrm{~m})$ 
Table 1. Experimental conditions.

\begin{tabular}{|c|c|c|c|c|c|c|c|}
\hline & $B(m)$ & $H(m)$ & $\dot{m}_{0}(\mathrm{~kg} / \mathrm{s})$ & $Z_{N}(\mathrm{~m})$ & $u_{0}(\mathrm{~m} / \mathrm{s})$ & $\dot{m}_{F}(\mathrm{~kg} / \mathrm{s})$ & $\dot{Q}(\mathrm{~kW})$ \\
\hline 1 & 0.2 & 0.5 & 0.0000 & 0.191 & 3.38 & 0.00257 & 56.1 \\
\hline 2 & 0.2 & 0.5 & 0.0290 & 0.159 & 4.34 & 0.00198 & 47.3 \\
\hline 3 & 0.2 & 0.5 & 0.0367 & 0.140 & 4.37 & 0.00178 & 43.4 \\
\hline 4 & 0.2 & 0.5 & 0.0528 & 0.137 & 4.11 & 0.0017 & 41.4 \\
\hline 5 & 0.3 & 0.5 & 0.0000 & 0.230 & 2.57 & 0.00149 & 34.5 \\
\hline 6 & 0.3 & 0.5 & 0.0306 & 0.188 & 3.08 & 0.0014 & 34.3 \\
\hline 7 & 0.3 & 0.5 & 0.0354 & 0.184 & 3.37 & 0.00162 & 39.5 \\
\hline 8 & 0.3 & 0.5 & 0.0458 & 0.184 & 3.21 & 0.00152 & 37.1 \\
\hline 9 & 0.5 & 0.5 & 0.0000 & 0.201 & 1.52 & 0.00102 & 18.8 \\
\hline 10 & 0.5 & 0.5 & 0.0281 & 0.259 & 2.06 & 0.00115 & 22.9 \\
\hline 11 & 0.5 & 0.5 & 0.0352 & 0.244 & 2.04 & 0.00122 & 23.3 \\
\hline 12 & 0.5 & 0.5 & 0.0548 & 0.239 & 2.00 & 0.00122 & 23.4 \\
\hline 13 & 0.3 & 0.3 & 0.0000 & 0.138 & 2.39 & 0.00143 & 23.3 \\
\hline 14 & 0.3 & 0.3 & 0.0310 & 0.108 & 2.15 & 0.00127 & 21.6 \\
\hline 15 & 0.3 & 0.3 & 0.0374 & 0.089 & 2.56 & 0.00153 & 26.3 \\
\hline 16 & 0.3 & 0.3 & 0.0525 & 0.083 & 2.90 & 0.00162 & 28.0 \\
\hline 17 & 0.5 & 0.3 & 0.0000 & 0.141 & 1.64 & 0.00139 & 23.5 \\
\hline 18 & 0.5 & 0.3 & 0.0312 & 0.135 & 2.00 & 0.00127 & 23.2 \\
\hline 19 & 0.5 & 0.3 & 0.0387 & 0.125 & 2.34 & 0.00143 & 26.2 \\
\hline 20 & 0.5 & 0.3 & 0.0554 & 0.115 & 2.67 & 0.00153 & 28.2 \\
\hline
\end{tabular}

